\title{
Minimal scanning duration for producing individualized repetitive transcranial magnetic stimulation targets
}

Jinmei Sun ( $\sim$ sunjinmei0628@163.com )

First Affiliated Hospital of Anhui Medical University https://orcid.org/0000-0002-9295-2759

Rongrong Du

Anhui Medical University

Bing Zhang

Anhui Medical University

Qiang Hua

Anhui Medical University

Yingru Wang

Anhui Medical University

Yuanyuan Zhang

Anhui Medical University

Gong-Jun JI

First Affiliated Hospital of Anhui Medical University

Kongliang He

Anhui Mental Health Center: Fourth People's Hospital of Hefei

Kai Wang

First Affiliated Hospital of Anhui Medical University

\section{Research Article}

Keywords: Dorsolateral prefrontal cortex, Individualized target, Inferior parietal lobule, Minimal scanning duration, Repetitive transcranial magnetic stimulation

Posted Date: February 28th, 2022

DOI: https://doi.org/10.21203/rs.3.rs-1362330/v1

License: (c) (1) This work is licensed under a Creative Commons Attribution 4.0 International License.

Read Full License 


\section{Abstract}

Objective: This study aimed to determine the minimal scanning duration of functional magnetic resonance imaging (fMRI) for producing individualized repetitive transcranial magnetic stimulation (rTMS) targets that are superior to the group-level targets.

Materials and Methods: This study included 30 healthy subjects and 20 depressive patients with highsampled fMRI data (> $69 \mathrm{~min}$ ). We computed suboptimal targets by gradually increasing the scanning duration beginning at $6 \mathrm{~min}$. The target connectivity and spatial distance to the optimal target (based on the full-duration scanning data) were compared to an anatomically fixed target from a group analysis (termed as the group target). These analyses were repeated for healthy subjects and depressive patients, as well as for target masks in the dorsal prefrontal cortex (DLPFC) and inferior parietal lobule (IPL).

Results: As the scanning duration increased, the suboptimal targets gradually approached the optimal targets in the healthy subjects. Compared with the group targets, the suboptimal targets in the DLPFC showed higher connectivity strength after $10 \mathrm{~min}$ of data collection and shorter spatial distance after 40 min. Similar results were found in major depressive patients. In the IPL, the minimal scanning duration decreased to 6 and $8 \mathrm{~min}$ for connectivity strength and distance, respectively.

Conclusion: These findings provide an important reference for individualized target definition in terms of scanning duration, which may standardize connectivity-based personalized studies.

\section{Introduction}

Major depressive disorder (MDD) is the leading cause of disability around the world, and which severely influences psychosocial functioning and diminishes the quality of life (Friedrich, 2017; Malhi and Mann, 2018). As few as $30 \%$ of patients are remitted after initial pharmacotherapy, with an increasingly lower remission rate for subsequent treatment (Rush, 2007). Repetitive transcranial magnetic stimulation (rTMS) is a noninvasive brain stimulation technique. It has been validated as a safe and effective approach for depressive patients who do not respond to pharmacological therapies (2016; Cole et al., 2020). However, there is still considerable heterogeneity in its clinical response (Yesavage et al., 2018).

Many factors could contribute to this response in variability (Fregni et al., 2006; Lisanby et al., 2009; Liston et al., 2014) and, of these, the suboptimal targeting area of the rTMS has received particular attention (Fox et al., 2013; Herbsman et al., 2009; Johnson et al., 2013; Weigand et al., 2018). Currently, there are mainly three types of targeting approaches, including scalp-based measurements, anatomical targeting, and connectivity-guided targeting. Studies routinely identified stimulation sites at a point $5 \mathrm{~cm}$ anterior to the hand motor cortex along the scalp (the " $5 \mathrm{~cm}$ rule"), but differences in head size may lead to inconsistent sites across different subjects (George et al., 2010; Lefaucheur et al., 2014). Populationbased anatomical coordinates can increase the precision and intersession reliability of stimulation (Fitzgerald et al., 2009; Herwig et al., 2001). However, this anatomical "one-site-fits-all" approach does not consider the high functional heterogeneity between individuals (especially in the association cortex) 
(Cash et al., 2021b; Li et al., 2020; Mueller et al., 2013; Petrides and Pandya, 1999; Rajkowska and Goldman-Rakic, 1995). Therefore, an individual-specific, connectivity-guided approach may be necessary for realizing optimal targeting of rTMS. Multiple retrospective studies have indicated antidepressant outcomes were better if stimulation sites in the dorsolateral prefrontal cortex (DLPFC) displayed stronger negative functional connectivity (FC) with the subgenual cingulate cortex (SGC) (Cash et al., 2019; Fox et al., 2012). This suggests the DLPFC subregion which was most anti-correlated with the SGC can be a potential individualized target.

Reliable individual FC patterns are essential for individualized targeting, which can be achieved by increasing the scanning duration (Birn et al., 2013; Gratton et al., 2020). Defining the individualized optimal rTMS targets require reviewing several hours of functional MRI (fMRI) data (Gordon et al., 2017; Gratton et al., 2020). However, a large proportion of clinical studies did not collect sufficient data (usually 5-10 min) to compute individualized targets (Anderson et al., 2011; Cash et al., 2021a; Gratton et al., 2020). To date, whether these short-time collection-based individual targets (i.e., suboptimal targets) can identify personalized connectivity patterns remains unclear.

Therefore, in this study, we aimed to determine the minimal scanning duration of fMRI to produce individualized targets that were superior to the group targets (i.e., targets using anatomical locating). We computed suboptimal personalized targets by gradually increasing the scanning duration of fMRI. The connectivity and spatial distances to the optimal personalized target (based on the full-duration scanning data) were compared to an anatomically fixed target (termed as the group target) from a group analysis. These analyses were repeated for healthy subjects and depressive patients and in the target mask in the DLPFC. Considering that the inferior parietal lobule (IPL) was a promising individualized target for memory enhancement, we also used it as a target mask for analysis (Gao et al., 2021; Wang et al., 2014).

\section{Methods}

\subsection{Overview}

The study included two high-sampled fMRI data from healthy subjects and patients with MDD. The healthy subject data were downloaded from the Neuroimaging Informatics Tools and Resources Clearinghouse (Chen et al., 2015). The MDD data were included from the Anhui Mental Health Center (Hefei, China). This study protocol was reviewed and approved by the Medical Ethics Committee of Anhui Medical University. All participants provided informed, written consent before experiments.

\subsection{Participants and data acquisition}

The MRI data of 30 healthy subjects (ages $24 \pm 2$ years, 15 females) were collected through a GE MR750 3.0 Tesla scanner (GE Medical Systems, Waukesha, WI) at CCBD, Hangzhou Normal University. (Chen et al., 2015). Each participant underwent ten imaging scans over one month with one scan every 3 days. 
The fMRI data of 20 depressive individuals with a diagnosis of MDD (ages $22 \pm 7$ years, 13 females) were collected from a clinical trial before and following rTMS. Detailed inclusion and exclusion criteria are provided in the Supplementary Material. Treatment comprised 2 weeks of daily stimulation targeting the left DLPFC. Each patient underwent two scans within 1 month (eight sessions, each 8 min and $40 \mathrm{~s}$ for a total of $69 \mathrm{~min}$ and $20 \mathrm{~s}$ ). All functional and structural MRI datasets were obtained at the University of Science and Technology of China (Hefei, China) with a 3-T scanner (Discovery 750; GE Healthcare, Milwaukee, WI, USA). The specific scanning parameters for both healthy and depressive subjects, and steps for neuroimaging preprocessing were provided in the Supplementary Material.

\subsection{Computation of the DLPFC target \\ 2.3.1 DLPFC region of interest (ROI)}

The DLPFC ROI was defined as the combined extent of $20 \mathrm{~mm}$ radius spheres centered along the left hemisphere at BA9 (MNI coordinate: $-36,39,43$ ), BA46 (MNI coordinate: $-44,40,29)$, and the " $5 \mathrm{~cm}$ " TMS site (MNI coordinate: $-41,16,54)$ (Fox et al., 2013).

\subsubsection{SGC Seedmap}

To increase the signal-to-noise ratio, seedmap methodology was applied in this study (Cash et al., 2021b; Fox et al., 2013). Specifically, we first computed the SGC to whole-brain FC in the group with a large sample data ( $n=99$, ages $29 \pm 10$ years, 62 females, scanning parameters, and preprocessing were the same as depressive patients). The SGC seed was a sphere ROI with a $10 \mathrm{~mm}$ radius at $\mathrm{MNI}$ coordinates $(6,16,-10)$. Then, the robust connectivity pattern of the SGC was calculated by a one-sample t-test and adopted as a weighted seedmap (excluding the left DLPFC ROI). Finally, the seedmap was used as a weighted seed for subsequent connectivity analyses.

\subsubsection{Computation of target}

\section{Group target}

The group-level DLPFC target was calculated as the most negatively-correlated site with the SGC seedmap in the dataset of 99 healthy subjects.

\section{Optimal target}

At the individual level, we defined the individualized optimal target in the DLPFC by computing FC based on the full-duration data of the healthy subjects (100 $\mathrm{min}$ ) or depressive patients ( $69 \mathrm{~min})$. The most negatively correlated site with the SGC seedmap in the left DLPFC was defined as the optimal target. Data concatenation on depressive patients was justified based on the absence of significant pre-post differences in SGC-DLPFC connectivity (Details are provided in the Supplementary Material).

\section{Suboptimal target}


At the individual level, the suboptimal personalized target was computed as the most negatively correlated site with the SGC seedmap in the left DLPFC based on the data with different scanning durations. The scanning duration increased gradually from 6 to $99 \mathrm{~min}$ (healthy subjects) or 6 to $68 \mathrm{~min}$ (depressive patients) with 1-min step intervals.

The detailed calculation process of the IPL target was described in the Supplementary Material.

\section{Statistical Methods}

The advantage of the suboptimal target was illustrated by comparing it with the group target. We used the analysis procedure in the DLPFC as an example for describing convenience (Fig. 1). Specifically: 1) we computed an "optimal FC map" of the SGC seedmap based on the full-duration fMRI data. 2) The connectivity values of the suboptimal and group targets in this "optimal FC map" were extracted for statistical comparisons. 3) As mentioned above, there was only one group target, but multiple suboptimal targets corresponding to different scanning durations (from 6 min to 99 min with 1-min step intervals). 4) The suboptimal and group targets were then compared in two measures: their connectivity value in the "optimal FC map" and their Euclidean distance to the optimal target in MNI space (Cash et al., 2021b; Cash et al., 2019; Fox et al., 2012). The comparison was performed by paired t-tests and corrected by FDR $(P<0.05)$.

\section{Results}

\subsection{Targets in the DLPFC}

\subsubsection{Healthy subjects}

\section{FC to the SGC}

The optimal targets showed a significantly negative correlation with the SGC seedmap (mean $\pm \mathrm{SD}=$ $-0.36 \pm 0.01 ; t=-30, P<0.0001 ;$ Fig. $2 A)$. The group target was at $(-45,39,24)$ in MNI space (mean $\pm S D$ $=-0.21 \pm 0.02 ; t=-12, P<0.0001 ;$ Fig. $2 A)$. The connectivity strength of suboptimal targets decreased with the increase in scanning duration (mean $\pm S D=-0.24 \pm 0.02$ to $-0.36 \pm 0.01$; Fig. $2 B$ ).

Particularly, the connectivity strength of the suboptimal target was significantly negative than that of the group target (Figs. $2 A-B ; P_{\text {corr }}<0.05$ ), as the scanning duration increased to 10 min (mean $\pm S D=-0.26$ \pm 0.02 ). Then, we used the connectivity strength at $10 \mathrm{~min}$ as a reference to search the next scanning duration that could produce significantly increased negative correlations. This comparison was repeated using updated references several times. Considering the practical feasibility in the clinic, the comparison was restricted to a $50 \mathrm{~min}$ duration. As a result, three scanning durations with statistically different connectivity strengths were found: $10 \mathrm{~min}$ (suboptimal) $>15 \mathrm{~min}$ (suboptimal) > $30 \mathrm{~min}$ (suboptimal) $\left(P_{\text {corr }}<0.05\right)$. 


\section{Distance to the optimal targets}

The distance from suboptimal targets to the optimal targets decreased as the scanning time duration increased from 6 to $99 \mathrm{~min}$ (mean $\pm \mathrm{SD}=28.01 \pm 3.39$ to $1.69 \pm 1.46 \mathrm{~mm}$; Fig. $2 \mathrm{C}$ ). Particularly, the distance from suboptimal targets to the optimal target was significantly shorter than that of the group target (mean $\pm S D=23.51 \pm 3.03 \mathrm{~mm}$; Fig. $2 \mathrm{~A}, \mathrm{C} ; \mathrm{P}_{\text {corr }}<0.05$ ) as the scanning duration increased to 40 $\min ($ mean $\pm S D=14.95 \pm 3.13 \mathrm{~mm})$.

\subsubsection{Depressive patients}

\section{FC to the SGC}

The optimal targets showed a significant negative correlation with the SGC seedmap (mean \pm SD $=-0.42$ $\pm 0.02 ; t=-22$, $P<0.0001$; Fig. $3 A)$. The group target was at $(-45,39,24)$ in MNI coordinates (mean $\pm S D$ $=-0.19 \pm 0.03 ; t=-6, P<0.0001 ; F i g .3 A)$. The connectivity strength of suboptimal targets in the depressive patients decreased as the scanning duration increased from 6 to $68 \mathrm{~min}$ (mean $\pm \mathrm{SD}=-0.28$ \pm 0.03 to $-0.42 \pm 0.02$; Fig. 3B).

The connectivity strength of suboptimal targets was significantly negative than that of group target (Figs. 3A-B; $P_{\text {corr }}<0.05$ ) as the scanning duration increased to 10 min (mean $\pm S D=-0.31 \pm 0.03$ ). We then searched the next scanning duration that could produce significantly more negative correlations. The results are as follows: $10 \mathrm{~min}$ (suboptimal) >14 $\mathrm{min}$ (suboptimal) $>17 \mathrm{~min}$ (suboptimal) $>28 \mathrm{~min}$ (suboptimal) $₫ 42$ min (suboptimal) $\left(P_{\text {corr }}<0.05\right)$.

\section{Distance to the optimal targets}

The distance from suboptimal targets to the optimal targets decreased as the scanning duration increased from 6 to $68 \mathrm{~min}$ (mean $\pm \mathrm{SD}=28.41 \pm 4.46$ to $0.21 \pm 0.21 \mathrm{~mm}$; Fig. $3 \mathrm{C}$ ). The distance from suboptimal targets to the optimal targets was significantly shorter than that of group target (mean $\pm \mathrm{SD}=$ $29.91 \pm 4.93 \mathrm{~mm}$; Figs. $\left.3 \mathrm{~A}, \mathrm{C} ; \mathrm{P}_{\text {corr }}<0.05\right)$ as the scanning duration increased to 44 min (mean $\pm \mathrm{SD}=$ $8.47 \pm 3.28 \mathrm{~mm})$.

\subsection{Targets of healthy subjects in the IPL}

\section{FC to the hippocampus}

The optimal targets showed a significant positive correlation with the hippocampal seedmap (mean \pm SD $=0.50 \pm 0.02 ; t=23, P<0.0001$; Fig. $4 \mathrm{~A})$. The group target was at $(-45,-66,27)$ in the MNI coordinates (mean $\pm S D=0.27 \pm 0.03 ; t=9, P<0.0001$; Fig. 4 A) The connectivity strength of suboptimal targets increased as the scanning duration increased from 6 to 99 min (mean $\pm S D=0.3841 \pm 0.04$ to $0.50 \pm 0.02$; Fig. 4B).

Notably, the connectivity strength of suboptimal targets calculated by a scanning duration of 6 min (mean $\pm S D=0.3841 \pm 0.04$ ) was already significantly more positive than that in group target $\left(P_{\text {corr }}<0.05\right.$; 
Figs. $4 A-B$ ). As a result, the connectivity strength calculated with a scanning duration of 33 min (mean \pm $S D=0.46 \pm 0.03)$ was significantly stronger than that at $6 \mathrm{~min}\left(P_{\text {corr }}<0.05\right)$.

\section{Distance to the optimal targets}

The distance from suboptimal targets to the optimal target decreased with an increased scanning duration (mean $\pm S D=7.63 \pm 0.91$ to $0.67 \pm 0.29$; Fig. 4 C). The distance from suboptimal targets to the optimal targets was significantly shorter than that in the group target (mean $\pm S D=9.13 \pm 0.75 ; P_{\text {corr }}<$ 0.05 ; Figs. $4 A, C$ ) as the scanning duration increased to 8 min (mean $\pm S D=7.39 \pm 0.84$ ). Next, we searched the next scanning duration that consistently could produce significantly shorter distances. The results were as follows: $8 \mathrm{~min}$ (suboptimal) $>18 \mathrm{~min}$ (suboptimal) $>34 \mathrm{~min}$ (suboptimal) $\left(P_{\text {corr }}<0.05\right)$.

\section{Discussion}

This study aimed to determine the minimal scanning durations of $\mathrm{fMRI}$ to produce individual targets that are superior to group targets. The analyses focused on the two heteromodal association cortices with high inter-subject variability, the DLPFC and IPL. In the DLPFC, we showed 10 and 40 min were the minimal scanning durations to identify individualized targets based on connectivity strength and distance, respectively. In the IPL, the minimal scanning duration was 6 and 8 min based on connectivity strength and distance, respectively. These findings suggested that the minimal scanning duration to produce targets with personalized connectivity feature may be inconsistent across the cortex.

The rTMS is widely accepted as an effective antidepressant treatment. Increasing evidence indicates that treatment response might be improved by stimulating areas more negatively correlated with the SGC and that are closer to personalized targets (Cash et al., 2021a; Cash et al., 2019; Fox et al., 2013). However, previous studies did not standardize the scanning duration. Our findings showed that the connectivity/spatial distance between suboptimal targets and optimal targets decreased gradually as scanning duration increased in the DLPFC. From the perspective of connectivity strength, 10 min was minimal scanning duration needed to calculate superior suboptimal targets in both healthy subjects and depressive patients. So far, many studies have met this standard (range from 10 to $25 \mathrm{~min}$ ) when investigating the relationship between DLPFC-SGC connectivity and treatment outcome. (Cash et al., 2021a; Fox et al., 2012; Siddiqi et al., 2019; Weigand et al., 2018). One individualized study applying multiple prolonged TMS for MDD achieved remarkable efficacy with only an 8 min scanning duration (Cole et al., 2020). Since $8 \mathrm{~min}$ is not long enough to identify the individualized target, we suggest the results of the study may be largely due to other influencing factors, such as high pulse doses, rather than an individualized targeting method (Li et al., 2020; Nettekoven et al., 2014). From the perspective of distance, 40 min and 44 min were the minimal scanning durations in the healthy subjects and patients, respectively. Previous research on distance mainly focused on the intraindividual reproducibility (Cash et al., 2021b; Ning et al., 2019) and, as a result, the acquisition time was recommended as 20 min. However, functional personalization is worthwhile only if the individualized target is superior to the group target. Therefore, we suggest that the scanning duration needs to be increased to about 40 min to meet the 
distance requirements. In general, the more negative connectivity appears easier to achieve than shorter distance, but both measures remain to be tested in clinical trials.

The human brain is characterized by robust interindividual variability in neuroanatomy and function (Frost and Goebel, 2012; Mueller et al., 2013). The personalized spatial location of resting-state networks varies more in patients compared with normal individuals (Dickie et al., 2018). Therefore, it is important to test the reproducibility of our results in patients before clinical application. Our study showed that the minimal scanning duration was very similar for depressive patients and healthy subjects. This finding demonstrated the similarity of DLPFC-SGC connectivity patterns in the two datasets, thus supporting the prior studies utilizing normative datasets to estimate the connectivity strength in depressive patients (Fox et al., 2012; Weigand et al., 2018). In addition, we further suggest that normative functional imaging be used to determine suitable scanning durations in the individualized treatment of patients with depression.

The left parietal cortex and its connectivity with the hippocampus are involved in different degrees of memory function (Ciaramelli et al., 2020; Paz-Alonso et al., 2013). Several studies have applied rTMS to locations with the strongest FC with the hippocampus within the lateral parietal cortex to investigate the changes of associative memory in normal subjects (Gao et al., 2021; Hendrikse et al., 2020; Wang et al., 2020; Wang et al., 2014). Nevertheless, these results were inconsistent. Different scanning durations (range from 10 to $58 \mathrm{~min}$ ) in these studies were speculated as an important factor for the outcome variability. In this study, we found that the suboptimal targets in the IPL approached the optimal targets gradually as the scanning duration increased. The minimum scanning duration (6 min for connectivity strength and $8 \mathrm{~min}$ for distance) has been achieved in most studies (Freedberg et al., 2019; Gao et al., 2021; Wang et al., 2014). Therefore, the results indicated that scanning duration may not be the major factor for the variability in the previous studies. Our findings in the IPL were based on data of healthy subjects. It would be interesting to test the generalization of these findings in old subjects or patients with Alzheimer's disease, since the memory issues exhibited in these cohorts may also be alleviated by rTMS on individualized targets (Nilakantan et al., 2019; Velioglu et al., 2021).

Finally, several limitations of our investigation are worth mentioning. First, the sample size was not large enough. Second, our findings in the IPL were based on data of healthy subjects. It should be further validated in patients with memory impairment before being adopted in rTMS treatment studies. Third, the current paper is a technique development paper so the clinical validity of the scanning duration we recommended remains to be proven.

\section{Conclusion}

In this study, we identified minimal scanning duration for computing individualized rTMS targets in the two heteromodal association cortices, the DLPFC and IPL. Our findings suggested that the minimal scanning duration of personalized target identification may be inconsistent across the cortex but has high reproducibility in healthy subjects and patients. In summary, these findings provide an important 
reference in terms of scanning duration that may standardize connectivity-based individualized rTMS studies.

\section{Declarations}

Acknowledgements The authors thank the participants for taking part in this study and thank Information Science Laboratory Center of USTC for the measurement services.

Author Contributions: All authors designed and conducted the study, including data collection and data analysis. Kai Wang, Gong-Jun JI and Kongliang He designed the study. Jinmei Sun, Rongrong Du, Bing Zhang and Qiang Hua conducted the study. Yingru Wang and Yuanyuan Zhang conducted the statistical analyses. Jinmei Sun drafted the manuscript, which all authors critically revised. Kai Wang and Gong-Jun $\mathrm{JI}$ had complete access to the study data. All authors approved the final manuscript.

Funding Sources: This work was supported by the National Natural Science Foundation of China, Grant/Award Numbers: 31970979, 82090034, 81971689; Scientific Research Fund of Anhui Medical University, Grant/Award Numbers: 2019xkj199; the Collaborative Innovation Center of Neuropsychiatric Disorders and Mental Health of Anhui Province, Grant/Award Numbers: 2020xkjT05.

\section{Compliance with Ethical Standards}

Ethical approval This study protocol was reviewed and approved by the Medical Ethics Committee of Anhui Medical University.

Consent to Participate The research protocol was performed according to the Helsinki Declaration of 1975 and approved by the Medical Ethics Committee of Anhui Medical University. Written informed consent was obtained from all participants after full explanation of the procedures involved.

Consent to publish This manuscript has not been published elsewhere and the authors agree to publication in the journal.

\section{Conflict of Interest}

The authors declare that they have no competing interests.

\section{Availability of data and materials}

The data and materials that support the findings of this study are available from the corresponding author upon reasonable request.

\section{Code availability}

The code that supports the findings of this study are available from the corresponding author upon reasonable request. 


\section{References}

1. Repetitive Transcranial Magnetic Stimulation for Treatment-Resistant Depression: A Systematic Review and Meta-Analysis of Randomized Controlled Trials. Ont Health Technol Assess Ser, 16,1-66

2. Anderson, J. S., Ferguson, M. A., Lopez-Larson, M., \& Yurgelun-Todd, D. (2011). Reproducibility of single-subject functional connectivity measurements. AJNR Am J Neuroradiol, 32, 548-555

3. Birn, R. M., Molloy, E. K., Patriat, R., Parker, T., Meier, T. B., Kirk, G. R. ... Prabhakaran, V. (2013). The effect of scan length on the reliability of resting-state fMRI connectivity estimates. Neuroimage, 83, $550-558$

4. Cash, R. F. H., Cocchi, L., Lv, J., Fitzgerald, P. B., \& Zalesky, A. (2021a). Functional Magnetic Resonance Imaging-Guided Personalization of Transcranial Magnetic Stimulation Treatment for Depression. JAMA Psychiatry, 78, 337-339

5. Cash, R. F. H., Cocchi, L., Lv, J., Wu, Y., Fitzgerald, P. B., \& Zalesky, A. (2021b). Personalized connectivity-guided DLPFC-TMS for depression: Advancing computational feasibility, precision and reproducibility. Hum Brain Mapp

6. Cash, R. F. H., Zalesky, A., Thomson, R. H., Tian, Y., Cocchi, L., \& Fitzgerald, P. B. (2019). Subgenual Functional Connectivity Predicts Antidepressant Treatment Response to Transcranial Magnetic Stimulation: Independent Validation and Evaluation of Personalization. Biol Psychiatry, 86, e5-e7

7. Chen, B., Xu, T., Zhou, C., Wang, L., Yang, N., Wang, Z. ... Weng, X. C. (2015). Individual Variability and Test-Retest Reliability Revealed by Ten Repeated Resting-State Brain Scans over One Month. PLoS One, 10, e0144963

8. Ciaramelli, E., Burianová, H., Vallesi, A., Cabeza, R., \& Moscovitch, M. (2020). Functional Interplay Between Posterior Parietal Cortex and Hippocampus During Detection of Memory Targets and Nontargets. Front Neurosci, 14, 563768

9. Cole, E. J., Stimpson, K. H., Bentzley, B. S., Gulser, M., Cherian, K., Tischler, C. ... Williams, N. R. (2020). Stanford Accelerated Intelligent Neuromodulation Therapy for Treatment-Resistant Depression. Am J Psychiatry, 177, 716-726

10. Dickie, E. W., Ameis, S. H., Shahab, S., Calarco, N., Smith, D. E., Miranda, D. ... Voineskos, A. N. (2018). Personalized Intrinsic Network Topography Mapping and Functional Connectivity Deficits in Autism Spectrum Disorder. Biol Psychiatry, 84, 278-286

11. Fitzgerald, P. B., Hoy, K., McQueen, S., Maller, J. J., Herring, S., Segrave, R. ... Daskalakis, Z. J. (2009). A randomized trial of $\mathrm{rTMS}$ targeted with MRI based neuro-navigation in treatment-resistant depression. Neuropsychopharmacology, 34, 1255-1262

12. Fox, M. D., Buckner, R. L., White, M. P., Greicius, M. D., \& Pascual-Leone, A. (2012). Efficacy of transcranial magnetic stimulation targets for depression is related to intrinsic functional connectivity with the subgenual cingulate. Biol Psychiatry, 72, 595-603

13. Fox, M. D., Liu, H., \& Pascual-Leone, A. (2013). Identification of reproducible individualized targets for treatment of depression with TMS based on intrinsic connectivity. Neuroimage, 66, 151-160 
14. Freedberg, M., Reeves, J. A., Toader, A. C., Hermiller, M. S., Voss, J. L., \& Wassermann, E. M. (2019). Persistent Enhancement of Hippocampal Network Connectivity by Parietal rTMS Is Reproducible. eNeuro, 6

15. Fregni, F., Marcolin, M. A., Myczkowski, M., Amiaz, R., Hasey, G., Rumi, D. O. ... Pascual-Leone, A. (2006). Predictors of antidepressant response in clinical trials of transcranial magnetic stimulation. Int J Neuropsychopharmacol, 9, 641-654

16. Friedrich, M. J. (2017). Depression Is the Leading Cause of Disability Around the World. Jama, 317, 1517

17. Frost, M. A., \& Goebel, R. (2012). Measuring structural-functional correspondence: spatial variability of specialised brain regions after macro-anatomical alignment. Neuroimage, 59, 1369-1381

18. Gao, X., Hua, Q., Du, R., Sun, J., Hu, T., Yang, J. ... Wang, K. (2021). Associative memory improvement after 5 days of magnetic stimulation: A replication experiment with active controls. Brain Res, 1765, 147510

19. George, M. S., Lisanby, S. H., Avery, D., McDonald, W. M., Durkalski, V., Pavlicova, M. ... Sackeim, H. A. (2010). Daily left prefrontal transcranial magnetic stimulation therapy for major depressive disorder: a sham-controlled randomized trial. Arch Gen Psychiatry, 67, 507-516

20. Gordon, E. M., Laumann, T. O., Gilmore, A. W., Newbold, D. J., Greene, D. J., Berg, J. J. ... Dosenbach (2017). N.U.F. Precision Functional Mapping of Individual Human Brains. Neuron, 95, 791-807.e797

21. Gratton, C., Kraus, B. T., Greene, D. J., Gordon, E. M., Laumann, T. O., Nelson, S. M. ... Petersen, S. E. (2020). Defining Individual-Specific Functional Neuroanatomy for Precision Psychiatry. Biol Psychiatry, 88, 28-39

22. Hendrikse, J., Coxon, J. P., Thompson, S., Suo, C., Fornito, A., Yücel, M., \& Rogasch, N. C. (2020). Multiday rTMS exerts site-specific effects on functional connectivity but does not influence associative memory performance. Cortex, 132, 423-440

23. Herbsman, T., Avery, D., Ramsey, D., Holtzheimer, P., Wadjik, C., Hardaway, F. ... Nahas, Z. (2009). More lateral and anterior prefrontal coil location is associated with better repetitive transcranial magnetic stimulation antidepressant response. Biol Psychiatry, 66, 509-515

24. Herwig, U., Schönfeldt-Lecuona, C., Wunderlich, A. P., von Tiesenhausen, C., Thielscher, A., Walter, H., \& Spitzer, M. (2001). The navigation of transcranial magnetic stimulation. Psychiatry Res, 108, 123131

25. Johnson, K. A., Baig, M., Ramsey, D., Lisanby, S. H., Avery, D., McDonald, W. M. ... Nahas, Z. (2013). Prefrontal rTMS for treating depression: location and intensity results from the OPT-TMS multi-site clinical trial. Brain Stimul, 6, 108-117

26. Lefaucheur, J. P., André-Obadia, N., Antal, A., Ayache, S. S., Baeken, C., Benninger, D. H. ... Paulus, W. (2014). \& Garcia-Larrea, L. Evidence-based guidelines on the therapeutic use of repetitive transcranial magnetic stimulation (rTMS). Clin Neurophysiol, 125, 2150-2206

27. Li, C. T., Cheng, C. M., Chen, M. H., Juan, C. H., Tu, P. C., Bai, Y. M. ... Su, T. P. (2020). Antidepressant Efficacy of Prolonged Intermittent Theta Burst Stimulation Monotherapy for Recurrent Depression 
and Comparison of Methods for Coil Positioning: A Randomized, Double-Blind, Sham-Controlled Study. Biol Psychiatry, 87, 443-450

28. Lisanby, S. H., Husain, M. M., Rosenquist, P. B., Maixner, D., Gutierrez, R., Krystal, A. ... George, M. S. (2009). Daily left prefrontal repetitive transcranial magnetic stimulation in the acute treatment of major depression: clinical predictors of outcome in a multisite, randomized controlled clinical trial. Neuropsychopharmacology, 34, 522-534

29. Liston, C., Chen, A. C., Zebley, B. D., Drysdale, A. T., Gordon, R., Leuchter, B. ... Dubin, M. J. (2014). Default mode network mechanisms of transcranial magnetic stimulation in depression. Biol Psychiatry, 76, 517-526

30. Malhi, G. S., \& Mann, J. J. (2018).Depression. Lancet, 392,2299-2312

31. Mueller, S., Wang, D., Fox, M. D., Yeo, B. T., Sepulcre, J., Sabuncu, M. R. ... Liu, H. (2013). Individual variability in functional connectivity architecture of the human brain. Neuron, 77, 586-595

32. Nettekoven, C., Volz, L. J., Kutscha, M., Pool, E. M., Rehme, A. K., Eickhoff, S. B. ... Grefkes, C. (2014). Dose-dependent effects of theta burst rTMS on cortical excitability and resting-state connectivity of the human motor system. $J$ Neurosci, 34, 6849-6859

33. Nilakantan, A. S., Mesulam, M. M., Weintraub, S., Karp, E. L., VanHaerents, S., \& Voss, J. L. (2019). Network-targeted stimulation engages neurobehavioral hallmarks of age-related memory decline. Neurology, 92, e2349-e2354

34. Ning, L., Makris, N., Camprodon, J. A., \& Rathi, Y. (2019). Limits and reproducibility of resting-state functional MRI definition of DLPFC targets for neuromodulation. Brain Stimul, 12, 129-138

35. Paz-Alonso, P. M., Gallego, P., \& Ghetti, S. (2013). Age differences in hippocampus-cortex connectivity during true and false memory retrieval. $J$ Int Neuropsychol Soc, 19, 1031-1041

36. Petrides, M., \& Pandya, D. N. (1999). Dorsolateral prefrontal cortex: comparative cytoarchitectonic analysis in the human and the macaque brain and corticocortical connection patterns. Eur $\mathrm{J}$ Neurosci, 11, 1011-1036

37. Rajkowska, G., \& Goldman-Rakic, P. S. (1995). Cytoarchitectonic definition of prefrontal areas in the normal human cortex: I. Remapping of areas 9 and 46 using quantitative criteria. Cereb Cortex, 5 , 307-322

38. Rush, A. J. (2007). Limitations in efficacy of antidepressant monotherapy. J Clin Psychiatry, 68(Suppl 10), 8-10

39. Siddiqi, S. H., Trapp, N. T., Shahim, P., Hacker, C. D., Laumann, T. O., Kandala, S. ... Brody, D. L. (2019). Individualized Connectome-Targeted Transcranial Magnetic Stimulation for Neuropsychiatric Sequelae of Repetitive Traumatic Brain Injury in a Retired NFL Player. J Neuropsychiatry Clin Neurosci, 31, 254-263

40. Velioglu, H. A., Hanoglu, L., Bayraktaroglu, Z., Toprak, G., Guler, E. M., Bektay, M. Y. ... Yulug, B. (2021). Left lateral parietal rTMS improves cognition and modulates resting brain connectivity in patients with Alzheimer's disease: Possible role of BDNF and oxidative stress. Neurobiol Learn Mem, 180, 107410 
41. Wang, H., Jin, J., Cui, D., Wang, X., Li, Y., Liu, Z., \& Yin, T. (2020). Cortico-Hippocampal Brain Connectivity-Guided Repetitive Transcranial Magnetic Stimulation Enhances Face-Cued Word-Based Associative Memory in the Short Term. Front Hum Neurosci, 14, 541791

42. Wang, J. X., Rogers, L. M., Gross, E. Z., Ryals, A. J., Dokucu, M. E., Brandstatt, K. L. ... Voss, J. L. (2014). Targeted enhancement of cortical-hippocampal brain networks and associative memory. Science, 345, 1054-1057

43. Weigand, A., Horn, A., Caballero, R., Cooke, D., Stern, A. P., Taylor, S. F. ... Fox, M. D. (2018). Prospective Validation That Subgenual Connectivity Predicts Antidepressant Efficacy of Transcranial Magnetic Stimulation Sites. Biol Psychiatry, 84, 28-37

44. Yesavage, J. A., Fairchild, J. K., Mi, Z., Biswas, K., Davis-Karim, A., Phibbs, C. S. ... George, M. S. (2018). Effect of Repetitive Transcranial Magnetic Stimulation on Treatment-Resistant Major Depression in US Veterans: A Randomized Clinical Trial. JAMA Psychiatry, 75, 884-893

\section{Figures}

\section{Figure 1}

Schematic of the experimental design. We used the analysis procedure in the DLPFC as an example. (A) Identification of the group target. (B) At the individual level, individualized targets were defined as the most negatively correlated sites with the SGC seedmap in the left DLPFC. Suboptimal individualized targets were calculated based on the data from 6 to 99 min scanning duration with 1-min step intervals. Optimal individualized targets were calculated based on the full-duration time scanning data (100 min). (C) One individualized "optimal FC map" of the SGC seedmap was calculated based on the full-duration time scanning data. To identify the minimal scanning duration, the suboptimal and group targets were compared in two measures: connectivity strength extracted from the "optimal FC map" and distance to the optimal target. 


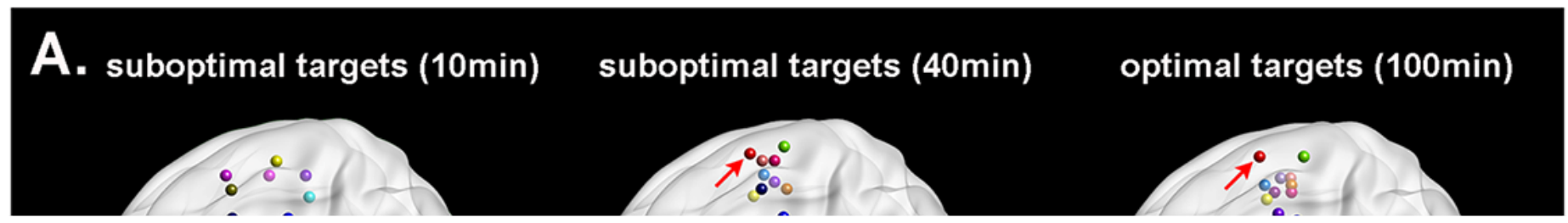

\section{Figure 2}

Comparisons of the group and suboptimal targets under the different measures of healthy subjects in the DLPFC. (A) Locations of group targets (bigger blue sphere), suboptimal targets at 10 and 40 min, and optimal targets. The red arrow points to the same subject at different scanning durations. (B) The mean connectivity strength of suboptimal targets (solid red line) was significantly more negative than that of the group target (blue line) at $10 \mathrm{~min}\left({ }^{*} \mathrm{P}_{\text {corr }}<0.05\right)$. (C) The mean distance from suboptimal (solid red line) targets to optimal targets was significantly shorter than that of the group target (blue line) at 40 min $\left({ }^{*} P_{\text {corr }}<0.05\right)$. 


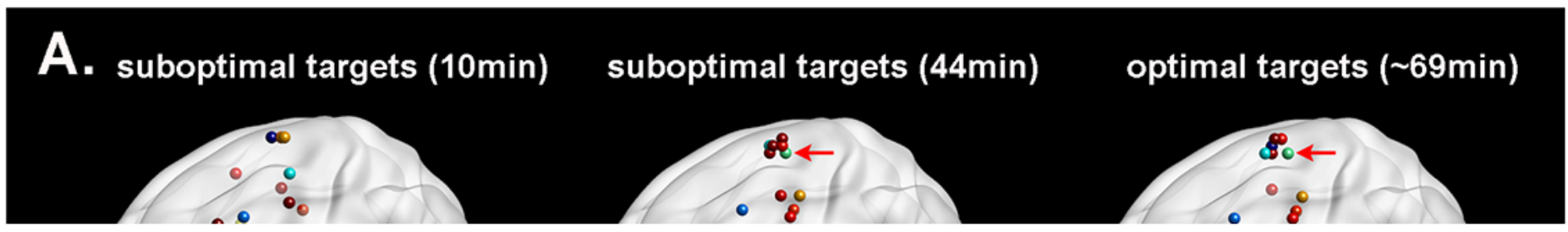

\section{Figure 3}

Comparisons of the group and suboptimal targets under the different measures of depressive patients in the DLPFC. (A) Locations of group targets (bigger blue sphere), suboptimal targets at 10 and 44 min, and optimal targets. The red arrow points to the same subject at different scanning duration. (B) The mean connectivity strength of suboptimal targets (solid red line) was significantly more negative than that of the group target (blue line) at $10 \mathrm{~min}\left({ }^{*} \mathrm{P}_{\text {corr }}<0.05\right)$. (C) The mean distance from suboptimal targets (solid red line) to optimal targets was significantly shorter than that of the group target (blue line) at 44 $\min \left({ }^{*} P_{\text {corr }}<0.05\right)$. 


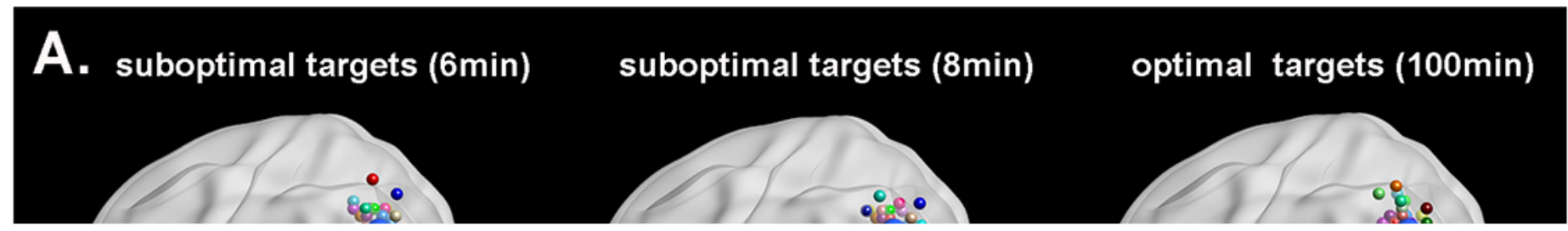

\section{Figure 4}

Comparisons of group and suboptimal targets under the different measures of healthy subjects in the IPL. (A) Locations of group targets (bigger blue sphere), suboptimal targets at 6 and 8 min, and optimal targets. The red arrow points to the same subject at different scanning durations. (B) The mean connectivity strength of suboptimal targets was significantly stronger than that of the group target ( ${ }^{*} \mathrm{P}_{\text {corr }}$ $<0.05$ ) at $6 \mathrm{~min}$. (C) The mean distance from suboptimal targets (solid red line) to optimal targets was significantly shorter that of the group target (blue line) at 8 min $\left({ }^{\star} P_{\text {corr }}<0.05\right)$.

\section{Supplementary Files}

This is a list of supplementary files associated with this preprint. Click to download.

- AuthorChecklist11682.pdf

- SupplementaryMaterial.docx 